\title{
Loss of quantum coherence through scattering off virtual black holes
}

\author{
S.W. Hawking ${ }^{a}$ and Simon F. Ross ${ }^{b}$ \\ ${ }^{a}$ Department of Applied Mathematics and Theoretical Physics \\ University of Cambridge, Silver St., Cambridge CB3 9EW \\ hawking@damtp.cam.ac.uk \\ ${ }^{b}$ Department of Physics, University of California \\ Santa Barbara, CA 93106 \\ sross@cosmic.physics.ucsb.edu
}

July 25, 2013

DAMTP/R-97/21

UCSB-TH-97-08

\begin{abstract}
In quantum gravity, fields may lose quantum coherence by scattering off vacuum fluctuations in which virtual black hole pairs appear and disappear. Although it is not possible to properly compute the scattering off such fluctuations, we argue that one can get useful qualitative results, which provide a guide to the possible effects of such scattering, by considering a quantum field on the $C$ metric, which has the same topology as a virtual black hole pair. We study a scalar field on the Lorentzian $C$ metric background, with the scalar field in the analytically-continued Euclidean vacuum state. We find that there are a finite number of particles at infinity in this state, contrary to recent claims made by Yi. Thus, this state is not determined by data at infinity, and there is loss of quantum coherence in this semi-classical calculation.
\end{abstract}




\section{Introduction}

The possible loss of quantum coherence is one of the most exciting topics in quantum gravity. Recent work on D-branes has encouraged those that believe that the evaporation of black holes is a unitary process without loss of quantum coherence. It has been shown that collections of strings attached to D-branes with the same mass and gauge charges as nearly extreme black holes have a number of internal states that is the same function of the mass and gauge charges as $e^{A / 4 G}$, where $A$ is the area of the horizon of the black hole $[1,2,3]$. They also seem to radiate various types of scalar particles $[4,5]$ at the same rate as the corresponding black holes. However, the D-brane calculations are valid only for weak coupling, at which string loops can be neglected. But at these weak couplings, the D-branes are definitely not black holes: there are no horizons, and the topology of spacetime is that of flat space. One can foliate such a spacetime with a family of nonintersecting surfaces of constant time. One can then evolve forward in time with the Hamiltonian and get a unitary transformation from the initial state to the final state. A unitary transformation would be a one to one mapping from the initial Hilbert space to the final Hilbert space. This would imply that there was no loss of information or quantum coherence.

To get something that corresponds to a black hole, one has to increase the string coupling constant until it becomes strong. This means that string loops can no longer be neglected. However, it is argued that for gauge charges that correspond to extreme, or near extreme black holes, the number of internal states will be protected by nonrenormalization theorems, and will remain the same. It is argued that there's no sign of a discontinuity as one increases the coupling, and therefore that the evolution should remain unitary. However, there's a very definite discontinuity when event horizons form: the Euclidean topology of spacetime will change from that of flat space, to something non-trivial. The change in topology will mean that any vector field that agrees with time translations at infinity, will necessarily have zeroes in the interior of the spacetime. In turn, this will mean that one cannot foliate spacetime with a family of time surfaces. If one tries, the surfaces will intersect at the zeroes of the vector field. One therefore cannot use the Hamiltonian to get a unitary evolution from an initial state to a final state. But if the evolution is not unitary, there will be loss of quantum coherence. An initial state that is a pure quantum state can evolve to a quantum state that is mixed. Another way of saying this is that the superscattering operator that maps initial density matrices to final density matrices will not factorise into the product of an $S$ matrix and its adjoint [6]. This will happen because the zeroes of the time translation vector field indicate that there will be horizons in the Lorentzian section. Quantum states on such a background are not completely determined by their asymptotic behavior, which is the necessary and sufficient condition for the superscattering operator to factorise.

One cannot just ignore topology and pretend one is in flat space. The recent progress 
in duality in gravitational theories is based on non-trivial topology. One considers small perturbations about different vacuums of the product form $M^{4} \times B$, and shows that one gets equivalent Kaluza-Klein theories. But if one can have small perturbations about product metrics, one should also consider larger fluctuations that change the topology from the product form. Indeed, such non-product topologies are necessary to describe pair creation or annihilation of solitons like black holes or p-branes.

It is often claimed that supergravity is just a low energy approximation to the fundamental theory, which is string theory. However, the recent work on duality seems to be telling us that string theory, p-branes and supergravity are all on a similar footing. None of them is the whole picture; instead, they are valid in different, but overlapping, regions. There may be some fundamental theory from which they can all be derived as different approximations. Or it may be that theoretical physics is like a manifold that can't be covered by a single coordinate patch. Instead, we may have to use a collection of apparently different theories that are valid in different regions, but which agree on the overlaps. After all, we know from Goedel's theorem that even arithmetic can't be reduced to a single set of axioms. Why should theoretical physics be different?

Even if there is a single formulation of the underlying fundamental theory, we don't have it yet. What is called string theory has a good loop expansion, but it is only perturbation theory about some background, generally flat space, so it will break down when the fluctuations become large enough to change the topology. Supergravity, on the other hand, is better at dealing with topological fluctuations, but it will probably diverge at some high number of loops. Such divergences don't mean that supergravity predicts infinite answers. It is just that it cannot predict beyond a certain degree of accuracy. But in that, it is no different from perturbative string theory. The string loop perturbation series almost certainly does not converge, but is only an asymptotic expansion. This means that higher order loop corrections get smaller at first. But after a certain order, the loop corrections will get bigger again. Thus at finite coupling, the string perturbation series will have only limited accuracy.

We shall take the above as justification for discussing loss of quantum coherence in terms of general relativity or supergravity, rather than D-branes and strings. One might expect that loss of quantum coherence could occur not only in the evaporation of macroscopic black holes, but on a microscopic level as well, because of topological fluctuations in the metric that can be interpreted as closed loops of virtual black holes [7]. Particles could fall into these virtual black holes, which would then radiate other particles. The emitted particles would be in a mixed quantum state because the presence of the black hole horizons will mean that a quantum state will not be determined completely by its behavior at infinity. It is with such loss of coherence through scattering off virtual black holes that this paper is concerned. Our primary intention is not to provide a rigorous demonstration that quantum coherence is lost, but rather to explore the effects that will arise, assuming that the semi-classical calculations are accurate, and it is lost. 
In $d$ dimensions, a single black hole has a Euclidean section with topology $S^{d-2} \times R^{2}$. As has been seen in studies of black hole pair creation, a real or virtual loop of black holes has Euclidean topology $S^{d-2} \times S^{2}-\{$ point , where the point has been sent to infinity by a conformal transformation. For simplicity, we shall consider $d=4$, but the treatment for higher $d$ would be similar.

On the manifold $S^{2} \times S^{2}-$ \{point one should consider Euclidean metrics that are asymptotic to flat space at infinity. Such metrics can be interpreted as closed loops of virtual black holes. Because they are off shell, they need not satisfy any field equations. They will contribute to the path integral, just as off shell loops of particles contribute to the path integral and produce measurable effects. The effect that we shall be concerned with for virtual black holes is loss of quantum coherence. This is a distinctive feature of such topological fluctuations that distinguishes them from ordinary unitary scattering, which is produced by fluctuations that do not change the topology.

One can calculate scattering in an asymptotically Euclidean metric on $S^{2} \times S^{2}-$ \{point\}. One then weights with $\exp (-I)$ and integrates over all asymptotically Euclidean metrics. This would give the full scattering with all quantum corrections. However, one can neither calculate the scattering in a general metric, nor integrate over all metrics. Instead, what we shall do in the next two sections is point out some qualitative features of the scattering in general metrics, that indicate that quantum coherence is lost. We shall then illustrate the effects of loss of quantum coherence and obtain an estimate of their magnitude by calculating the scattering in a specific metric on $S^{2} \times S^{2}-\{$ point , the $C$ metric. It is sufficient to show that quantum coherence is lost in some metrics in the path integral, because the integral over other metrics cannot restore the quantum coherence lost in our examples.

\section{Lorentzian section}

We don't have much intuition for the behavior of Euclidean Green functions or their effect on scattering. However, if the Euclidean metric has a hypersurface orthogonal killing vector, it can be analytically continued to a real Lorentzian metric, in which it is much easier to see what is happening. We shall therefore consider scattering in such metrics.

The Lorentzian section of an asymptotically Euclidean metric which has topology $S^{2} \times S^{2}-\{$ point $\}$ will contain a pair of black holes that accelerate away from each other and go off to infinity. One might think that this is not very physical, but it is no different from a closed loop of a particle like an electron. Closed particle loops are really defined in Euclidean space. If one analytically continues them to Minkowski space, one gets a particle anti-particle pair accelerating away from each other. Any topologically non-trivial asymptotically Euclidean metric will appear to have solitons accelerating to 
infinity in the Lorentzian section, but this does not mean that there are actual black holes at infinity, any more than there are runaway electrons and positrons with a virtual electron loop. One can regard the use of the Lorentzian metric, with its black holes accelerating to infinity, as just a mathematical trick to evaluate the scattering on the Euclidean solution.

To understand the structure of these accelerating black hole metrics, it is helpful to draw Penrose diagrams. Start with the Penrose diagram for Rindler space with the left and right acceleration horizons, $H_{a l}$ and $H_{a r}$, and past and future null infinity, $\mathcal{I}^{-}$and $\mathcal{I}^{+}$(see Figure 1). A uniformly accelerated particle moves on a world line that goes out to $\mathcal{I}^{-}$and $\mathcal{I}^{+}$at the points where they intersect the acceleration horizons. One now replaces the accelerating particle and the similar accelerating particle on the other side with black holes. Thus, one replaces the regions of Rindler space to the right and left of the accelerating world lines with intersecting black hole horizons. It turns out that the two accelerating black holes are just the two sides of the same three dimensional wormhole, so one has to identify the two sides of the Penrose diagram, and the Penrose diagram will look like the one in Figure 2. At first sight it looks as if one has lost half of $\mathcal{I}^{-}$and $\mathcal{I}^{+}$, but that is because this Penrose diagram applies only on the axis. One can get a better idea of the causal structure near infinity from Figure 3 , in which a conformal transformation has been used to make $\mathcal{I}^{+}$into a cylinder $S^{2} \times R^{1}$, with the null generators lying in the $R^{1}$ direction. The hypersurface orthogonal Killing vector of the Euclidean metric that allows continuation to a Lorentzian metric will be a boost Killing vector in the accelerating black hole metric and it will have two fixed points $q$ and $r$ on $\mathcal{I}^{+}$, lying on generators $\lambda$ and $\lambda^{\prime}$ respectively. The past light cones of $q$ and $r$ minus the generators $\lambda$ and $\lambda^{\prime}$ form the acceleration horizons. Thus one can see that nearly every null geodesic outside the black hole horizons goes out to $\mathcal{I}^{+}$in the region to the future of both acceleration horizons. The exceptions are the null geodesics that are exactly in the boost direction, which intersect the generators $\lambda$ and $\lambda^{\prime}$. We shall ignore $\lambda$ and $\lambda^{\prime}$ as a set of measure zero on $\mathcal{I}^{+}$, and a number of the statements we shall make will be valid modulo this set of measure zero.

\section{Quantum state}

The analytically continued Euclidean Green functions will define a vacuum state $|0\rangle_{E}$ which is the analogue of the so-called Hartle Hawking state [8] for a static black hole. The Euclidean quantum state can be characterized by saying that positive frequency means positive frequency with respect to the affine parameters on the horizons. In the accelerating black hole metrics there are two kinds of horizons, black hole and acceleration. Each kind of horizon consists of two intersecting null hypersurfaces, which we shall refer to as left and right, as in Figure 2. In choosing a Cauchy surface for the 


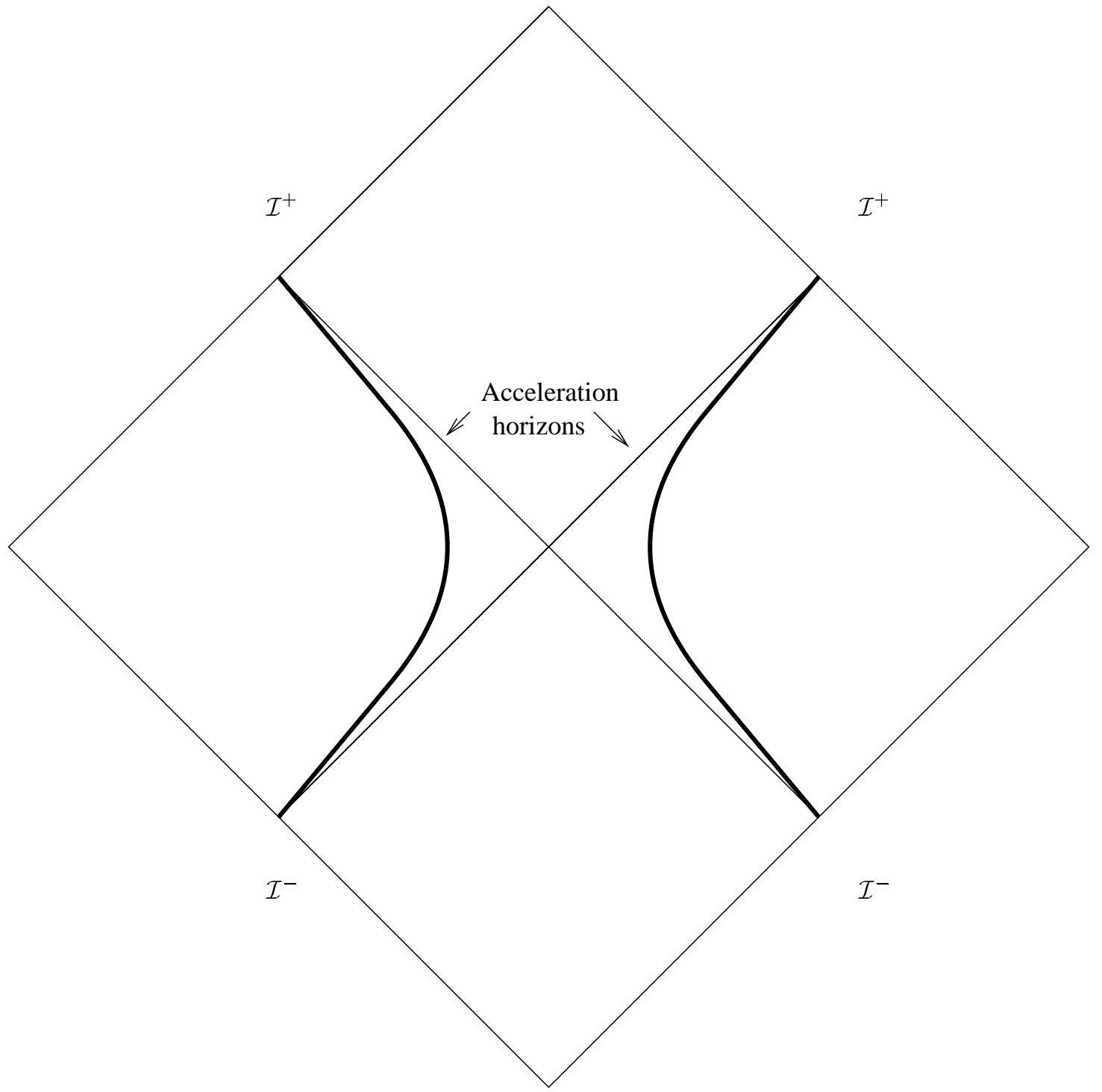

Figure 1: The causal structure of Rindler space, with a pair of accelerating particles depicted. 
spacetime (modulo a set of measure zero), we break the symmetry between left and right, and choose say the left acceleration horizon and the right black hole horizon. The quantum state defined by positive frequency with respect to the affine parameters on these horizons is the same as the quantum state defined by the other choice of horizons.

Another Cauchy surface in the future (again modulo a set of measure zero) is formed by $\mathcal{I}^{+}$and the future parts of the black hole horizons $H_{b l}^{+}$and $H_{b r}^{+}$, as in Figure 4. There is a natural notion of positive frequency on $\mathcal{I}^{+}$. On the black hole horizons the concept of positive frequency is less well defined. One could use Rindler time, but in any case, what one observes on $\mathcal{I}^{+}$is independent of the choice of positive frequency on the black hole horizons.

The quantum state of a field $\phi$ on this background metric will be determined by data on either of these Cauchy surfaces. This means that the Hilbert space $\mathcal{H}$ of quantum fields on this background metric will be isomorphic to the tensor products of the Fock spaces on their components:

$$
\begin{aligned}
\mathcal{H} & =\mathcal{F}_{H_{a l}} \otimes \mathcal{F}_{H_{b r}} \\
& =\mathcal{F}_{\mathcal{I}^{+}} \otimes \mathcal{F}_{H_{b l}^{+}} \otimes \mathcal{F}_{H_{b r}^{+}}
\end{aligned}
$$

The vacuum state defined by the Euclidean Green functions is the product of the vacuum states of the Fock spaces for the left acceleration horizon and right black hole horizon;

$$
|0\rangle_{E}=|0\rangle_{H_{a l}}|0\rangle_{H_{b r}}
$$

However, because of frequency mixing, the Euclidean quantum state won't be the product of the Fock vacuum states on $\mathcal{I}^{+}$and the future black hole horizons. Rather it will be a state containing pairs of particles. Both members of the pair may go out to $\mathcal{I}^{+}$, or both may fall into the holes, or one go out to $\mathcal{I}^{+}$and one fall in.

Equation (1) shows that quantum field theory on an accelerating black hole background does not satisfy the asymptotic completeness condition that the Hilbert space of the quantum fields on the background is isomorphic to the asymptotic Hilbert space of states on $\mathcal{I}^{+}$. Asymptotic completeness is the necessary and sufficient condition for scattering of quantum fields on the background to be unitary [6]. Thus there will be loss of quantum coherence. What happens is that to calculate the probability of observing particles at $\mathcal{I}^{+}$, one has to trace out over all possibilities on the future black hole horizons. This reduces the Euclidean quantum state to what appears to be a mixed quantum state described by a density matrix.

In a recent pair of papers $[9,10]$, Yi argued that the Euclidean quantum state in the Ernst metric would contain no radiation at infinity. The Ernst metric is similar to the metrics we are considering. However, in the explicit calculation that we carry out in the $C$ metric, we find that there is indeed radiation at infinity. What's wrong with Yi's argument? As he was working with the Ernst metric, which isn't asymptotically 
flat, he wasn't able to study the radiation at infinity directly. He therefore assumed that if there was no radiation on the acceleration horizon, there would be no radiation at infinity. But if we evolve some state forward from one of the acceleration horizons to $\mathcal{I}^{+}$, part of the state can fall into the future black hole horizon. Therefore, there can be a non-trivial Bogoliubov transformation between the acceleration horizon and infinity, and Yi's assumption is incorrect.

The Euclidean quantum state $|0\rangle_{E}$ will be time symmetric, and so will contain both incoming and outgoing radiation. Unlike the Euclidean state for static black holes, there won't be radiation to infinity at a steady rate for an infinite time. Instead, the radiation will be peaked around the points $q$ and $r$ where the acceleration horizons intersect $\mathcal{I}^{+}$. The radiation will die off at early and late times and the total energy radiated will be finite.

Is this the appropriate quantum state? In the case of a static black hole, one usually imposes the boundary condition that there is no incoming radiation on $\mathcal{I}^{-}$. This means that one has to subtract the incoming radiation from the Euclidean state to give what is called the Unruh state. This is singular on the past horizon, but that doesn't matter, as one normally replaces this region of the metric with the metric of a collapsing body. The energy for the steady rate of outgoing radiation comes from a slow decrease of the mass of the black hole formed by the collapse. However, in the case of a virtual black hole loop, there is no collapse process to remove the singularities on the past horizons of the black holes or supply the energy of the outgoing radiation. Therefore, we should study the Euclidean vacuum state, in which the energy of the outgoing radiation is supplied by the incoming radiation on $\mathcal{I}^{-}$.

Our view therefore is that integrating over gauge equivalent virtual black hole metrics will cause the amplitude to be zero unless the energy of the outgoing particle or particles is matched by particles with the same energy falling in. One might object that one would never have exactly the combination of incoming particles that corresponded to the quantum state obtained from the Euclidean green functions. However, the Euclidean quantum state will appear to be a mixed quantum state on $\mathcal{I}^{-}$which contains every possible combination of incoming particles. One can choose one of these combinations as an initial pure quantum state that is incident on the virtual black hole loop. The final quantum state will then be that part of the Euclidean quantum state on $\mathcal{I}^{+}$that has the same energy, momentum and angular momentum as the incoming state. Because of the trace over the future black hole horizon states, the final state on $\mathcal{I}^{+}$will be mixed. Such an evolution from pure to mixed states can be described by a superscattering operator $\$$ rather than an $S$ matrix [6].

The dominant contribution will presumably come from virtual black hole loops of Planck size. The cross section for a low energy particle to fall into a Planck size static black hole is very low unless the particle is spin 0 or $1 / 2$ [11]. In the case of spin $1 / 2$, the probability of emission will be reduced because the Fermi-Dirac factor $(\exp (\omega / T)+1)^{-1}$ 
tends to 1 at low $\omega$ while $(\exp (\omega / T)-1)^{-1}$ tends to $T / \omega$. This suggests the effects of virtual black holes will be small except for scalar particles. In this paper we shall therefore do a scattering calculation for scalar particles in the $C$ metric. This doesn't really qualify as a virtual black hole metric, because it has conical singularities on the axis, although one can interpret these as cosmic strings. We study the $C$ metric because it has the same topological structure as a virtual black hole pair, but it has the great advantage that one can calculate the scattering, because the wave equation separates.

\section{$4 \quad C$ metric}

The charged $C$ metric solution is [12]

$$
d s^{2}=A^{-2}(x-y)^{-2}\left[G(y) d t^{2}-G^{-1}(y) d y^{2}+G^{-1}(x) d x^{2}+G(x) d \varphi^{2}\right],
$$

where

$$
G(\xi)=\left(1+r_{-} A \xi\right)\left(1-\xi^{2}-r_{+} A \xi^{3}\right)=-r_{+} r_{-} A^{2}\left(\xi-\xi_{1}\right)\left(\xi-\xi_{2}\right)\left(\xi-\xi_{3}\right)\left(\xi-\xi_{4}\right) .
$$

The gauge potential is

$$
A_{\varphi}=q\left(x-\xi_{3}\right)
$$

where $q^{2}=r_{+} r_{-}$. We define $m=\left(r_{+}+r_{-}\right) / 2$. We constrain the parameters so that $G(\xi)$ has four roots, which we label by $\xi_{1} \leq \xi_{2}<\xi_{3}<\xi_{4}$. To obtain the right signature, we restrict $x$ to $\xi_{3} \leq x \leq \xi_{4}$, and $y$ to $-\infty<y \leq x$. The inner black hole horizon lies at $y=\xi_{1}$, the outer black hole horizon at $y=\xi_{2}$, and the acceleration horizon at $y=\xi_{3}$. The axis $x=\xi_{4}$ points towards the other black hole, and the axis $x=\xi_{3}$ points towards infinity. Spatial infinity is at $x=y=\xi_{3}$, null and timelike infinity at $x=y \neq \xi_{3}$. This metric describes a pair of oppositely-charged black holes accelerating away from each other, although the coordinate system used in (3) only covers the neighborhood of one of the black holes.

To avoid having a conical singularity between the two black holes, we choose

$$
\Delta \varphi=\frac{4 \pi}{\left|G^{\prime}\left(\xi_{4}\right)\right|} .
$$

This implies that there will be a conical deficit along $x=\xi_{3}$, with deficit angle

$$
\delta=2 \pi\left(1-\left|\frac{G^{\prime}\left(\xi_{3}\right)}{G^{\prime}\left(\xi_{4}\right)}\right|\right) .
$$

Physically, we imagine that this represents a cosmic string of mass per unit length $\mu=\delta / 8 \pi$ along $x=\xi_{3}$. At large spatial distances, that is, as $x, y \rightarrow \xi_{3}$, the $C$ metric 
(3) reduces to flat space with conical deficit $\delta$ in accelerated coordinates. The $C$ metric also reduces to flat space if we set $r_{+}=r_{-}=0$. It reduces to a single static black hole if we set $A=0$ [13]. The limit $r_{+} A \ll 1$ is referred to as the point-particle limit, as in this limit the black hole is small on the scale set by the acceleration.

The $C$ metric was shown to be asymptotically flat in [14]. This is a considerable advantage over, say, the Ernst metric, as it means we will have a well-defined notion of $\mathcal{I}$, and we can study the radiation at infinity directly. If we neglect the axis $x=\xi_{3}$, all observers will intersect the acceleration horizon before reaching infinity, and the causal structure of the solution is roughly speaking given by the Penrose diagram shown in Figure 2. However, the metric is not spherically symmetric, so this diagram is not a true picture of the whole spacetime. We will refer to the left and right acceleration horizons as $H_{a l}$ and $H_{a r}$, and to the left and right outer black hole horizons as $H_{b l}$ and $H_{b r}$. Further, the future and past halves of each horizon will be denoted by superscripts \pm . Hopefully the diagram clarifies the meaning of this notation.

We will only discuss the behavior at future null infinity. As the metric is timesymmetric, the discussion of past null infinity will be identical. We can conformally compactify the $C$ metric by using a conformal factor $\Omega=A(x-y)$. The conformally rescaled metric is

$$
\tilde{d s}^{2}=\Omega^{2} d s^{2}=G(y) d t^{2}-G^{-1}(y) d y^{2}+G^{-1}(x) d x^{2}+G(x) d \varphi^{2} .
$$

Null infinity is the surface $\Omega=0$, that is, $x=y$ (more precisely, its maximal extension; the coordinate system of (8) misses the generator on which the other black hole intersects $\left.\mathcal{I}^{+}[14]\right)$. The induced metric on $\mathcal{I}^{+}$is

$$
\tilde{d}_{\mathcal{I}}^{2}=G(y)\left(d t^{2}+d \varphi^{2}\right) .
$$

Note that, at null infinity, $t$ is a spatial coordinate. The normal to $\mathcal{I}^{+}$is

$$
n^{a}=\tilde{\nabla}^{a} \Omega=2 A G(y) \partial_{y} .
$$

We see that $t$ and $\varphi$ are constant along the orbits of $n^{a}$, which are the generators of $\mathcal{I}^{+}$, so they are good coordinates on the manifold of orbits of $\mathcal{I}^{+}$. It is convenient to define new coordinates $\theta, \eta$ where

$$
\frac{d \theta}{\sin \theta}=\frac{\left|G^{\prime}\left(\xi_{4}\right)\right|}{2} d t, \quad \eta=\frac{\left|G^{\prime}\left(\xi_{4}\right)\right|}{2} \varphi
$$

(so $\Delta \eta=2 \pi$ ). We also make a further conformal rescaling with a conformal factor $\Omega^{\prime}=\left|G^{\prime}\left(\xi_{4}\right)\right| \sin \theta / 2 G^{1 / 2}(y)$, so that

$$
\check{d}_{\mathcal{I}}^{2}=\Omega^{\prime 2} \tilde{d}_{\mathcal{I}}^{2}=d \theta^{2}+\sin ^{2} \theta d \eta^{2} .
$$




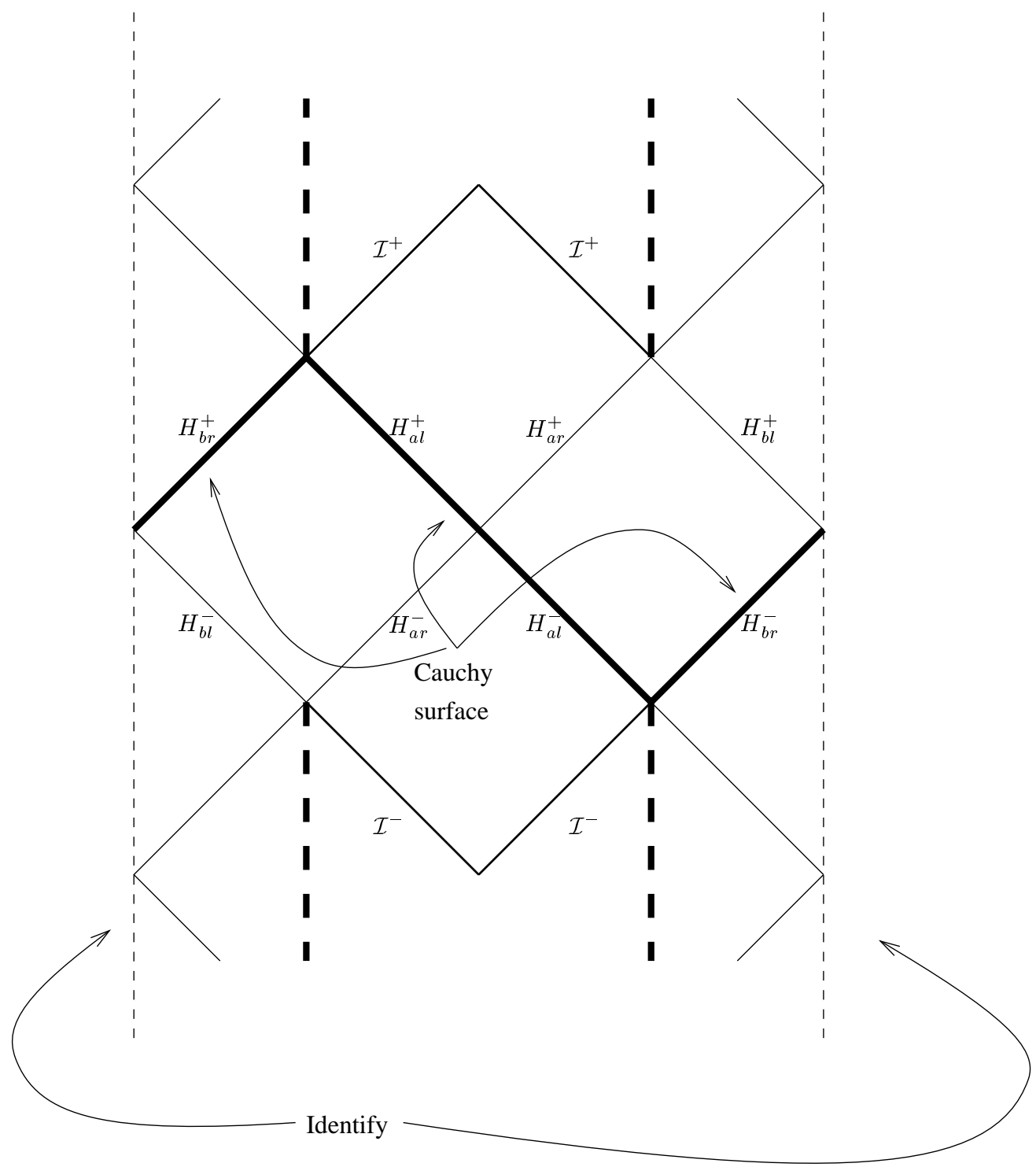

Figure 2: A Penrose diagram for the $C$ metric, neglecting the axis $x=\xi_{3}$. The heavy dashed lines are singularities, and the surfaces $\mathcal{I}^{ \pm}$are boundaries of the spacetime. A Cauchy surface $\mathcal{C}$ for the region outside the inner black hole horizons constructed from one black hole horizon and one acceleration horizon is shown. 


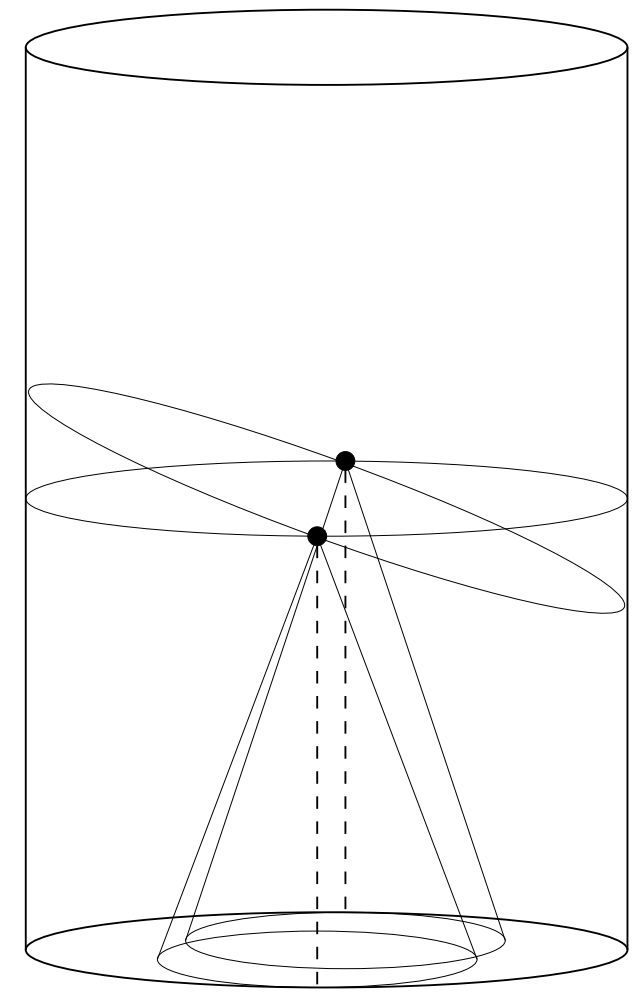

Figure 3: The structure of $\mathcal{I}^{+}$in the conformal gauge (12). The two points are where the black holes intersect $\mathcal{I}^{+}$, and their past light cones are the acceleration horizons. Two of the $\theta, \eta$ cross-sections are pictured. The dashed lines represent the conical deficits in the metric (3); they are not part of $\mathcal{I}^{+}$.

In this conformal gauge, an affine parameter along the generators of $\mathcal{I}^{+}$is

$$
\tilde{r}=\frac{\left|G^{\prime}\left(\xi_{4}\right)\right| \sin \theta}{4 A} \int \frac{d y}{G(y)^{3 / 2}} .
$$

It is also useful to define another coordinate

$$
r=\int \frac{d y}{G(y)^{3 / 2}}
$$

which labels the $\theta, \eta$ cross-sections. The structure of $\mathcal{I}^{+}$in the conformal gauge (12) is depicted in Figure 3. In this conformal gauge, $\mathcal{I}^{+}$is divergence-free, and $\theta, \eta$ are coordinates on the manifold of generators of $\mathcal{I}^{+}$, so we can see that $\mathcal{I}^{+}$has topology $S^{2} \times R$.

We can obtain the Euclidean section of the $C$ metric by setting $t=i \tau$ in (3). To make the Euclidean metric positive definite, we need to restrict the range of $y$ to $\xi_{2} \leq y \leq \xi_{3}$. 
There are then potentially conical singularities at $y=\xi_{2}$ and $y=\xi_{3}$, which have to be eliminated. We can avoid having a conical singularity at $y=\xi_{3}$ by taking $\tau$ to be periodic with period

$$
\Delta \tau=\beta=\frac{4 \pi}{G^{\prime}\left(\xi_{3}\right)}
$$

In this paper, we assume the black holes are non-extreme, that is, $\xi_{1}<\xi_{2}$. We can then only avoid having a conical singularity at $y=\xi_{2}$ by taking the two horizons to have the same temperature, so that both conical singularities can be removed by the same choice of $\Delta \tau$. This implies

$$
\xi_{2}-\xi_{1}=\xi_{4}-\xi_{3}
$$

The Euclidean section has topology $S^{2} \times S^{2}-\{p t\}$. This Euclidean section can be used to study the pair creation of black holes by breaking cosmic strings $[15,16,17]$. However, we want to use it simply to determine the appropriate vacuum state on the Lorentzian section. Since the black hole and acceleration horizon have the same temperature on the Euclidean section, the analytic continuation will give Green's functions which are thermal with temperature $1 / \beta$ with respect to the time parameter $t$ in the Lorentzian section.

The region of the spacetime outside the inner horizon of the black holes is globally hyperbolic. Consider a Cauchy surface for this region which is made up of one black hole horizon and one acceleration horizon (say the left acceleration horizon and the right black hole horizon), as pictured in Figure 2. As explained earlier, the Hilbert space is isomorphic to the tensor product of the Fock spaces on the two horizons (1). Positive frequency on the Fock spaces is defined with respect to the affine parameter along the horizon. The state we wish to study is the analytically-continued Euclidean vacuum state $|0\rangle_{E}$ given in (2).

In the next section, we will describe the solution of the scalar wave equation on the $C$ metric background. We then use this to calculate the Bogoliubov coefficients in the subsequent section.

\section{Scalar Wave Equation}

We consider a minimally-coupled massless neutral scalar field, so the wave equation is just $\square \phi=0$. One of the great advantages of considering the $C$ metric is that this equation separates. It is easy to see this if we observe that the $C$ metric is a solution of the vacuum Einstein-Maxwell equations, and hence $R=0$. The minimally coupled equation

above is therefore equivalent to the conformally-invariant equation $\square \phi-\frac{1}{6} R \phi=0$. But in solving this latter equation, we are free to make conformal transformations. In particular, we can transform to the conformal gauge (8), in which this equation takes 
the form

$$
\frac{1}{G(y)} \partial_{t} \partial_{t} \tilde{\phi}-\partial_{y}\left[G(y) \partial_{y} \tilde{\phi}\right]+\partial_{x}\left[G(x) \partial_{x} \tilde{\phi}\right]+\frac{1}{G(x)} \partial_{\varphi} \partial_{\varphi} \tilde{\phi}+\frac{1}{6}\left[\partial_{x}^{2} G(x)-\partial_{y}^{2} G(y)\right] \tilde{\phi}=0,
$$

where because of the conformal rescaling, $\tilde{\phi}=\phi / A(x-y)$. Thus we see that if we use the ansatz

$$
\phi=A(x-y) e^{i \omega t} e^{i m \varphi} \nu(x) \gamma(y),
$$

then we get two second-order ODEs for $\nu(x)$ and $\gamma(y)$,

$$
\partial_{x}\left[G(x) \partial_{x} \nu(x)\right]-\frac{1}{G(x)} m^{2} \nu(x)+\left[\frac{1}{6} \partial_{x}^{2} G(x)+D\right] \nu(x)=0
$$

and

$$
\partial_{y}\left[G(y) \partial_{y} \gamma(y)\right]+\frac{1}{G(y)} \omega^{2} \gamma(y)+\left[\frac{1}{6} \partial_{y}^{2} G(y)+D\right] \gamma(y)=0,
$$

where $D$ is a separation constant, and $G(\xi)$ is given in (4). Note that $\varphi$ is a periodic coordinate with period $4 \pi /\left|G^{\prime}\left(\xi_{4}\right)\right|$. Thus $m=m_{0}\left|G^{\prime}\left(\xi_{4}\right)\right| / 2$, where $m_{0}$ is an integer. We assume, without loss of generality, that it is positive.

One way to rewrite these equations that offers some further insight is to define new coordinates

$$
z=\int \frac{d y}{G(y)}, \quad \chi=\int \frac{d x}{G(x)},
$$

which have the advantage that $\partial_{z}=G(y) \partial_{y}, \partial_{\chi}=G(x) \partial_{x}$. Note that the integral for $z$ in (21) diverges as we approach a horizon, as $G(y) \rightarrow 0$ at the horizons. Thus, $-\infty<z<\infty$ only covers the region between two of the horizons; similarly, $\xi_{3}<x<\xi_{4}$ is mapped to $-\infty<\chi<\infty$. We can write $(19,20)$ as

$$
\begin{gathered}
\partial_{\chi}^{2} \nu(x(\chi))-m^{2} \nu(x(\chi))+V_{e f f}(\chi) \nu(x(\chi))=0 \\
\partial_{z}^{2} \gamma(y(z))+\omega^{2} \gamma(y(z))+V_{e f f}(z) \gamma(y(z))=0 .
\end{gathered}
$$

That is, (20) reduces to the one-dimensional wave equation with effective potential $V_{\text {eff }}(z)$, which is given by

$$
V_{e f f}(z)=G(y(z))\left[\frac{1}{6} \partial_{y}^{2} G(y(z))+D\right] .
$$

There is a similar expression for $V_{\text {eff }}(\chi)$. It is not possible to invert (21) to obtain $y(z)$ explicitly, but we can make some observations. Near the horizons, $G(y) \rightarrow 0$, and thus the effective potential becomes unimportant, so $\gamma(y) \sim e^{ \pm i \omega z}$. Similarly, near $x=\xi_{3}, \xi_{4}$, $\nu(x) \sim e^{ \pm m \chi}$. Obviously, for physically-interesting solutions, we must have $\nu(x) \sim e^{-m|\chi|}$ as $\chi \rightarrow \pm \infty$. 
We can rewrite the metric (3) in terms of these coordinates:

$$
d s^{2}=A^{-2}(x-y)^{-2}\left[G(y)\left(d t^{2}-d z^{2}\right)+G(x)\left(d \chi^{2}+d \varphi^{2}\right)\right],
$$

where by $x, y$ we mean $x(\chi), y(z)$. This coordinate system evidently only covers the region between two of the horizons (or between the acceleration horizon and infinity). That is, there is a coordinate system like this for each of the diamond-shaped regions in the Penrose diagram in Figure 2. We will therefore refer to these as the Rindlerian coordinates. We can now define null coordinates $u, v=t \pm z$. Since $z$ increases as we go from the acceleration horizon towards the black hole horizon, the $u$ and $v$ coordinates run as shown in Figure 4. Thus, $u$ is a (non-affine) parameter along $H_{a r}^{ \pm}$and $H_{b r}^{ \pm}$, while $v$ is a (non-affine) parameter along $H_{a l}^{ \pm}$and $H_{b l}^{ \pm}$. As is usual for bifurcate Killing horizons, these parameters are related to the affine parameters $U, V$ on the acceleration horizon by $u=\frac{1}{\kappa} \ln |U|, v=-\frac{1}{\kappa} \ln |V|$, where $\kappa=G^{\prime}\left(\xi_{3}\right) / 2$ is the common surface gravity of the two horizons.

These coordinates are useful for specifying boundary conditions near the black hole and the acceleration horizons, and we will see later that we can easily write down explicit forms for the positive-frequency wavefunctions on the horizons in terms of them. However, as we can't write $V_{\text {eff }}$ explicitly as a function of $z$, we can't solve the differential equations in this form.

If we return to the initial forms $(19,20)$ for the ODEs, we find that they can be considerably simplified. In the simplification, we will exploit the equal-temperature condition (16), which imposes an additional symmetry on the form of $G(\xi)$. If we make a coordinate transformation

$$
\hat{\xi}=\frac{2}{\left(\xi_{3}-\xi_{2}\right)}\left[\xi-\frac{1}{2}\left(\xi_{3}+\xi_{2}\right)\right],
$$

then

$$
G(\xi)=-\frac{\psi}{\zeta}\left(\hat{\xi}^{2}-\alpha^{2}\right)\left(\hat{\xi}^{2}-1\right),
$$

where

$$
\zeta=\frac{8}{r_{+} r_{-} A^{2}\left(\xi_{3}-\xi_{2}\right)^{3}}, \quad \alpha=\frac{\left(\xi_{4}-\xi_{1}\right)}{\left(\xi_{3}-\xi_{2}\right)}, \quad \psi=\frac{1}{2}\left(\xi_{3}-\xi_{2}\right) .
$$

Note that $\alpha>1, \zeta, \psi>0$, and that $\partial_{\hat{\xi}}=\psi \partial_{\xi}$. If $\hat{y}$ and $\hat{x}$ are defined in terms of $y$ and $x$ following (26), then the inner black hole horizon is at $\hat{y}=-\alpha$, the outer black hole horizon is at $\hat{y}=-1$, and the acceleration horizon is at $\hat{y}=1$, while the range of $\hat{x}$ is $1 \leq \hat{x} \leq \alpha$. In terms of these coordinates,

$$
\partial_{\xi}^{2} G(\xi)=-\frac{1}{\zeta \psi}\left[12 \hat{\xi}^{2}-2\left(1+\alpha^{2}\right)\right],
$$




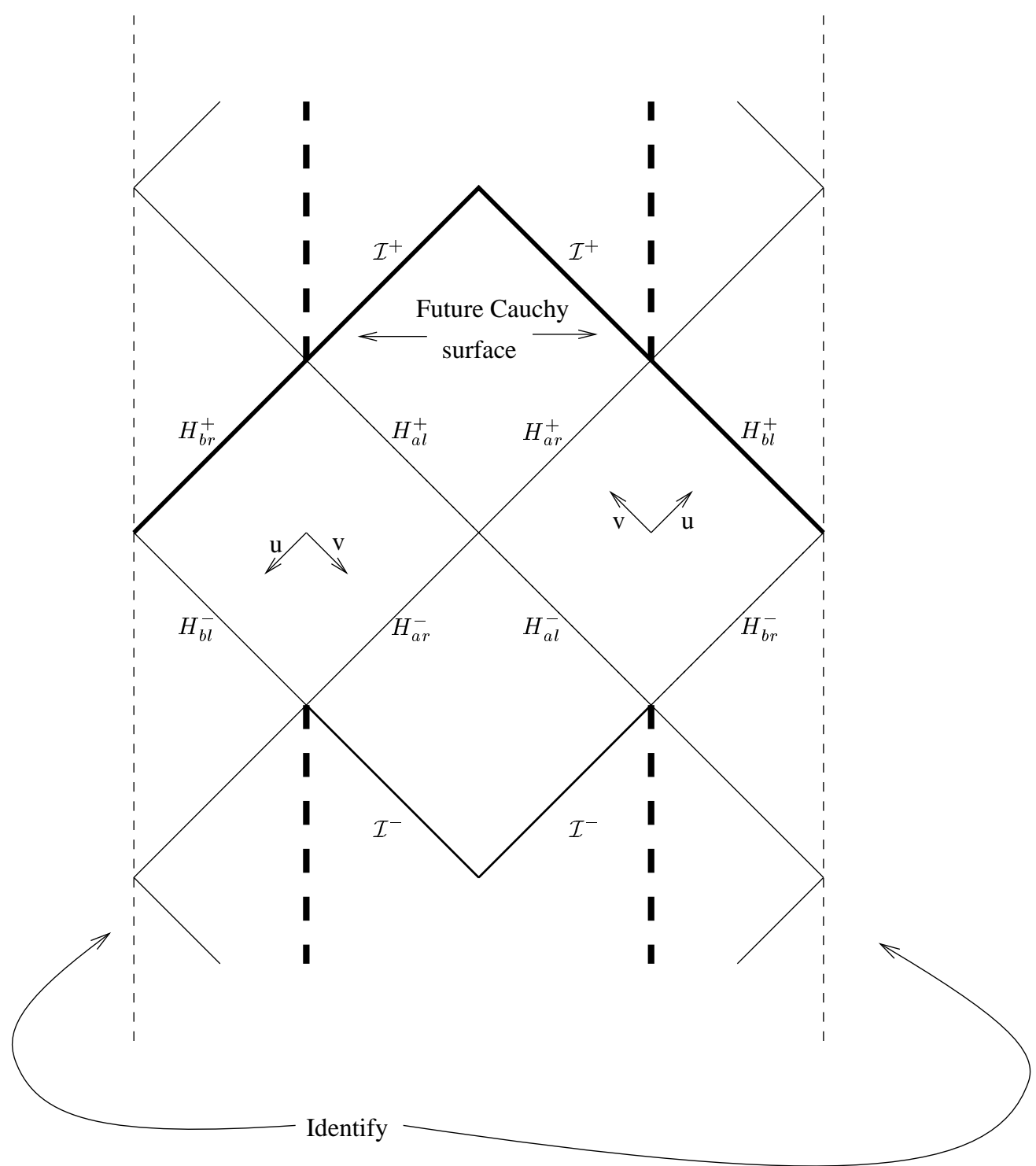

Figure 4: A Cauchy surface $\tilde{\mathcal{C}}$ for the region outside the inner black hole horizons constructed from $\mathcal{I}^{+}$and the future halves of the black hole horizons. The Rindlerian coordinates $u, v$ between the acceleration and outer black hole horizons are also shown. 
so it is convenient to define

$$
\beta_{D}^{2}=\frac{1}{6}\left(1+\alpha^{2}\right)+\frac{D \psi \zeta}{2}
$$

so that

$$
\frac{1}{6} \partial_{\xi}^{2} G(\xi)+D=-\frac{2}{\zeta \psi}\left(\hat{\xi}^{2}-\beta_{D}^{2}\right)
$$

We can now write $z$ explicitly;

$$
z=\int \frac{d y}{G(y)}=\frac{\zeta}{2\left(\alpha^{2}-1\right)}\left[\frac{1}{\alpha} \ln \left|\frac{\alpha+\hat{y}}{\alpha-\hat{y}}\right|+\ln \left|\frac{1-\hat{y}}{1+\hat{y}}\right|\right] .
$$

We can now see clearly that $z$ diverges at the event horizons $\hat{y}=-\alpha, \pm 1$. We can further see that $z \rightarrow-\infty$ as we approach $\hat{y}=-\alpha, 1$, the inner black hole and acceleration horizons, and $z \rightarrow \infty$ as we approach $\hat{y}=-1$, the outer black hole horizon. There is a similar explicit expression for $\chi$, and $\chi \rightarrow-\infty$ as we approach $\hat{x}=1$ and $\chi \rightarrow \infty$ as we approach $\hat{x}=\alpha$. The consideration of the form $(22,23)$ suggests a further simplifying transformation. If we set

$$
\hat{\nu}(\hat{x})=e^{m \chi} \hat{n}(\hat{x})=\left(\frac{\alpha+\hat{x}}{\alpha-\hat{x}}\right)^{\frac{\zeta m}{2 \alpha\left(\alpha^{2}-1\right)}}\left(\frac{\hat{x}-1}{1+\hat{x}}\right)^{\frac{\zeta m}{2\left(\alpha^{2}-1\right)}} \hat{n}(\hat{x})
$$

and

$$
\hat{\gamma}(\hat{y})=e^{-i \omega z} \hat{f}(\hat{y})=\left(\frac{\alpha+\hat{y}}{\alpha-\hat{y}}\right)^{\frac{-i \zeta \omega}{2 \alpha\left(\alpha^{2}-1\right)}}\left(\frac{1-\hat{y}}{1+\hat{y}}\right)^{\frac{-i \zeta \omega}{2\left(\alpha^{2}-1\right)}} \hat{f}(\hat{y}),
$$

then we can finally rewrite $(19,20)$ as

$$
\begin{aligned}
& \partial_{\hat{x}}\left[\left(\hat{x}^{2}-1\right)\left(\hat{x}^{2}-\alpha^{2}\right) \partial_{\hat{x}} \hat{n}(\hat{x})\right]-2 m \zeta \partial_{\hat{x}} \hat{n}(\hat{x})+2\left(\hat{x}^{2}-\beta_{D}^{2}\right) \hat{n}(\hat{x})=0, \\
& \partial_{\hat{y}}\left[\left(\hat{y}^{2}-1\right)\left(\hat{y}^{2}-\alpha^{2}\right) \partial_{\hat{y}} \hat{f}(\hat{y})\right]+2 i \omega \zeta \partial_{\hat{y}} \hat{f}(\hat{y})+2\left(\hat{y}^{2}-\beta_{D}^{2}\right) \hat{f}(\hat{y})=0 .
\end{aligned}
$$

This is the simplest form in which we can write these equations.

We have been able to simplify the form of the wave equation considerably. However, $(35,36)$ still have five regular singular points, at $\hat{\xi}= \pm 1, \pm \alpha, \infty$, so they can't be solved exactly. We will therefore need to use some further simplifying assumption in solving the wave equation. There is only one dimensionless parameter in the metric, $r_{+} A$, as the equal-temperature condition fixes $r_{-} A$ as a function of $r_{+} A$. Therefore we are driven to consider the point-particle limit $r_{+} A \ll 1$. In this limit, $\alpha \approx 1+4 r_{+} A$, and $\zeta \approx 8 r_{+} A \approx 2(\alpha-1)$. For reasons of convenience, we will use $(\alpha-1)$ as the small parameter. 


\section{Bogoliubov Transformations}

Having laid the groundwork, we can now define and evaluate the Bogoliubov coefficients. We can write the field operator $\phi$ in terms of annihilation and creation operators on the Hilbert spaces associated with the black hole and acceleration horizons:

$$
\phi=A(x-y) \Sigma_{l m} \int d \omega\left(f_{\omega l m}^{b} b_{\omega l m}^{b}+\bar{f}_{\omega l m}^{b} b_{\omega l m}^{b \dagger}+f_{\omega l m}^{a} b_{\omega l m}^{a}+\bar{f}_{\omega l m}^{a} b_{\omega l m}^{a \dagger}\right),
$$

where $f_{\omega l m}^{b}, f_{\omega l m}^{a}$ are sets of positive frequency modes which have non-zero support on the black hole and acceleration horizons respectively, $b_{\omega l m}^{b}, b_{\omega l m}^{a}$ are the particle annihilation operators, and $b_{\omega l m}^{b \dagger}, b_{\omega l m}^{a \dagger}$ are the particle creation operators. Here, positive frequency means with respect to the affine parameters $U, V$ on the horizons.

Following [18], we see that a suitable set of positive frequency states on the black hole horizon is

$$
f_{\omega l m}^{b}=\frac{N}{\left|1-e^{-2 \pi \omega / \kappa}\right|^{1 / 2}} e^{i m \varphi} \nu_{l m}(x)\left[g_{\omega}^{-}+e^{-\pi \omega / \kappa} g_{\omega}^{+}\right]
$$

where $\nu_{l m}$ is a solution of (19) with $D$ given by $\beta_{D}=1+2 l(l+1)$, and $g_{\omega}^{ \pm}$are functions which are non-zero on the future and past parts of the black hole horizon respectively, and which are positive frequency with respect to the Rindler parameter, that is, $g_{\omega}^{ \pm}=e^{-i \omega u}$. We know already that only a discrete set of values for $m$ are allowed, and we will see below that the same is true for $l$. We wish to normalize the modes so that $\left(f_{\omega l m}^{b}, f_{\omega^{\prime} l^{\prime} m^{\prime}}^{b}\right)=$ $\delta_{m m^{\prime}} \delta_{l l^{\prime}} \delta\left(\omega-\omega^{\prime}\right)$, which implies $|N|^{2}=1 /(4 \pi|\omega| \Delta \varphi)$. Note that although the positivefrequency solutions are labeled by a frequency $\omega$, they do not have a single frequency with respect to $U$, and the solutions are still wholly positive frequency with respect to $U$ when $\omega$ is negative. For this to be a complete set of positive-frequency solutions, we must allow $\omega$ to run over $-\infty<\omega<\infty$. One can write down a similar set of positive frequency solutions on the acceleration horizon.

In appendix $\mathrm{A}$, we consider $(35)$ with $(\alpha-1) \ll 1$, and we learn that, as we might have expected, there is a restriction on the form of the data on the black hole horizon. If we write $l=l_{0}+O(\alpha-1)$, then the solutions $\nu_{l m}(x)$ will only be regular at both of the axes $x=\xi_{3}, x=\xi_{4}$ if $l_{0}$ is an integer and $l_{0} \geq m_{0}$, where $m_{0}$ is the integer appearing in $m$. In the point-particle limit, the $x, \varphi$ section approaches spherical symmetry, so $l_{0}$ is the usual total angular momentum quantum number, while $m_{0}$ is the angular momentum with respect to the axis along which the black holes are accelerating.

We can also write the field operator in terms of modes which are positive frequency at infinity:

$$
\phi=A(x-y) \int d \omega d \theta_{0} d \eta_{0}\left(p_{\omega} a_{\omega}+\bar{p}_{\omega} a_{\omega}^{\dagger}+q_{\omega} c_{\omega}+\bar{q}_{\omega} c_{\omega}^{\dagger}\right)
$$

where $p_{\omega}$ are a set of modes with non-zero support on $\mathcal{I}^{+}$which are positive frequency with respect to $\tilde{r}$, and $a_{\omega}, a_{\omega}^{\dagger}$ are the corresponding annihilation and creation operators. 
The modes $q_{\omega}$ have non-zero support on the future black hole horizon, and $c_{\omega}, c_{\omega}^{\dagger}$ are the corresponding annihilation and creation operators. We won't bother to define these latter modes, as their form is irrelevant to the calculation of particle production on $\mathcal{I}^{+}$.

Following [19], we choose the positive frequency modes $p_{\omega}$ to have the form

$$
p_{\omega}=\frac{e^{-i \tilde{\omega} \tilde{r}}}{\sqrt{2 \pi} \tilde{\omega} \sin \theta_{0}} \delta\left(\theta-\theta_{0}\right) \delta\left(\eta-\eta_{0}\right)=\frac{e^{-i \omega r}\left|G^{\prime}\left(\xi_{4}\right)\right|}{\sqrt{2 \pi} 4 A \omega} \delta\left(\theta-\theta_{0}\right) \delta\left(\eta-\eta_{0}\right)
$$

on $\mathcal{I}^{+}$, in the conformal gauge where the metric on $\mathcal{I}^{+}$has the form (12). We define $\omega=\tilde{\omega}\left|G^{\prime}\left(\xi_{4}\right)\right| \sin \theta_{0} / 4 A$. Each mode is thus non-zero on one generator of $\mathcal{I}^{+}$, labeled by $\theta_{0}, \eta_{0}$, and has frequency $\tilde{\omega}$ with respect to the affine parameter along that generator. The complete set of positive frequency modes is given by $0 \leq \omega<\infty$. They are normalized so that $\left(p_{\omega}, p_{\omega}^{\prime}\right)=2 \tilde{\omega} \delta^{3}\left(\vec{k}-\vec{k}^{\prime}\right)$, where $\vec{k}$ is the three-momentum, and points in the direction $\left(\theta_{0}, \eta_{0}\right)$.

Since both sets of modes are complete bases for the space of solutions of the wave equation, we can write one in terms of the other. That is,

$$
f_{\omega^{\prime} l m}^{b}=\int d \tilde{\omega} d \theta_{0} d \eta_{0}\left(\alpha_{\omega \omega^{\prime} l m}^{b} p_{\omega}+\beta_{\omega \omega^{\prime} l m}^{b} \bar{p}_{\omega}+\text { terms involving } q_{\omega}\right),
$$

and similarly for $f_{\omega^{\prime} l m}^{a}$. If we substitute these expansion into (37), and require consistency with (39), then we find that

$$
a_{\omega}=\Sigma_{l m} \int d \omega^{\prime}\left(\alpha_{\omega \omega^{\prime} l m}^{b} b_{\omega^{\prime} l m}^{b}+\bar{\beta}_{\omega \omega^{\prime} l m}^{b} b_{\omega^{\prime} l m}^{b \dagger}+\alpha_{\omega \omega^{\prime} l m}^{a} b_{\omega^{\prime} l m}^{a}+\bar{\beta}_{\omega \omega^{\prime} l m}^{a} b_{\omega^{\prime} l m}^{a \dagger}\right) .
$$

The quantities $\alpha_{\omega \omega^{\prime} l m}^{b}, \beta_{\omega \omega^{\prime} l m}^{b}, \alpha_{\omega \omega^{\prime} l m}^{a}, \beta_{\omega \omega^{\prime} l m}^{a}$ are called the Bogoliubov coefficients. Since we know how the annihilation and creation operators which were defined on the horizons act on $|0\rangle_{E}$, to determine how the annihilation and creation operators defined at infinity act on $|0\rangle_{E}$, we just need to compute these coefficients.

The operator we are most interested in is the number operator $N_{\omega}=a_{\omega}^{\dagger} a_{\omega}$, which gives the number of particles in the mode $p_{\omega}$. In the state $|0\rangle_{E}$,

$$
\begin{aligned}
\left\langle 0\left|N_{\omega}\right| 0\right\rangle_{E} & =\Sigma_{l m l^{\prime} m^{\prime}} \int d \omega^{\prime} d \omega^{\prime \prime}\left(\beta_{\omega \omega^{\prime} l m}^{b} \bar{\beta}_{\omega \omega^{\prime \prime} l^{\prime} m^{\prime}}^{b}\left\langle 0\left|b_{\omega^{\prime} l m}^{b} b_{\omega^{\prime \prime} l^{\prime} m^{\prime}}^{b \dagger}\right| 0\right\rangle_{E}+\ldots\right) \\
& =\Sigma_{l m} \int_{-\infty}^{\infty} d \omega^{\prime}\left(\left|\beta_{\omega \omega^{\prime} l m}^{b}\right|^{2}+\left|\beta_{\omega \omega^{\prime} l m}^{a}\right|^{2}\right)
\end{aligned}
$$

where we have expanded $a_{\omega}$ by (42), and in the second line we have used the canonical commutation relations and the fact that $b_{\omega^{\prime} l m}^{b}|0\rangle_{E}=0, b_{\omega^{\prime} l m}^{a}|0\rangle_{E}=0$.

We should now calculate the Bogoliubov coefficients $\beta_{\omega \omega^{\prime} l m}^{b}$ and $\beta_{\omega \omega^{\prime} l m}^{a}$. However, it turns out to be quite difficult to calculate the latter coefficient. Therefore, we wish to argue that it is sufficient to calculate the contribution from the Bogoliubov coefficient associated with the black hole horizon $\beta_{\omega \omega^{\prime} l m}^{b}$; the other contribution should be similar. 
We broke the symmetry between the left and right horizons when we defined the Euclidean vacuum state, by defining it to be the product of the vacua of the Fock spaces for the left acceleration horizon and right black hole horizon. However, the vacuum state is in fact symmetric under left-right interchange. That is, it is also equal to the product of the vacua of the Fock spaces for the right acceleration horizon and left black hole horizon. Take the vacuum state on $\mathcal{C}$ and evolve it forward through the right Rindler diamond, from $H_{a l}^{-}$and $H_{b r}^{-}$to $H_{a r}^{+}$and $H_{b l}^{+}$. There will then be correlations between $H_{b r}^{+}$and $H_{a r}^{+}$, due to the correlations between the two halves of the black hole horizon in the Cauchy surface $\mathcal{C}$. Further, there are no correlations between $H_{b r}^{+}$and $H_{a l}^{+}$, because on $\mathcal{C}$, the state has no correlations between the black hole and acceleration horizons. Since the state is left-right symmetric, the correlations between the two halves of the acceleration horizon in the Cauchy surface $\mathcal{C}$ can therefore only give rise to correlations between $H_{b l}^{+}$and $H_{a l}^{+}$, and these correlations will be related to the ones coming from the black hole horizon. Both sets of correlations give rise to correlations between $\mathcal{I}^{+}$and the future black hole horizons, which give the particle creation, so the particle creation due to the acceleration horizon should just be the image under the left-right interchange of the particle creation due to the black hole horizon. This justifies our only calculating the latter contribution.

We now calculate $\beta_{\omega \omega^{\prime} l m}^{b}$. The modes $p_{\omega}, \bar{p}_{\omega}, q_{\omega}, \bar{q}_{\omega}$ are orthogonal, and

$$
\left(p_{\omega}, p_{\omega^{\prime}}\right)=2 \tilde{\omega} \delta^{3}\left(\vec{k}-\vec{k}^{\prime}\right)=\frac{2}{\tilde{\omega} \sin \theta_{0}} \delta\left(\eta_{0}-\eta_{0}^{\prime}\right) \delta\left(\theta_{0}-\theta_{0}^{\prime}\right) \delta\left(\tilde{\omega}-\tilde{\omega}^{\prime}\right) .
$$

Thus, we can use (41) to show

$$
\bar{\beta}_{\omega \omega^{\prime} l m}^{b}=\frac{\tilde{\omega} \sin \theta_{0}}{2}\left(p_{\omega}, \bar{f}_{\omega^{\prime} l m}^{b}\right)=\frac{2 A \omega}{\left|G^{\prime}\left(\xi_{4}\right)\right|}\left(p_{\omega}, \bar{f}_{\omega^{\prime} l m}^{b}\right) .
$$

To evaluate this inner product, we need to express both the modes as functions on the same Cauchy surface. We do this by evolving the mode $\bar{f}_{\omega^{\prime} l m}^{b}$ forwards from $\mathcal{C}$ to $\tilde{\mathcal{C}}$.

The propagation from $\mathcal{C}$ to $\tilde{\mathcal{C}}$ can be broken up into two stages: propagation through the right Rindler diamond, from $H_{a l}^{-}$and $H_{b r}^{-}$to $H_{a r}^{+}$and $H_{b l}^{+}$, and propagation through the future diamond, from $H_{a l}^{+}$and $H_{a r}^{+}$to $\mathcal{I}^{+}$. The initial data on $H_{b r}^{-}$is just the restriction of (38) to the past part of the black hole horizon, while $\bar{f}_{\omega^{\prime} l m}^{b}$ vanishes on $H_{a l}^{-}$. From the discussion of (23), we recall that at the acceleration and black hole horizons, $\gamma(y) \sim e^{ \pm i \omega z}$. Using this and the form (38) of the mode $f_{\omega^{\prime} l m}^{b}$, we find that the boundary conditions on $\gamma(y)$ are

$$
\gamma(y)=e^{-i \omega z}+C_{R} e^{i \omega z}
$$

at the black hole horizon $z \rightarrow \infty$, and

$$
\gamma(y)=C_{T} e^{-i \omega z}
$$


at the acceleration horizon $z \rightarrow-\infty$, where $C_{R}$ and $C_{T}$ are constants which remain to be determined. In appendix $\mathrm{B}$, we solve (36) with these boundary conditions in the limit $r_{+} A \ll 1$, assuming $\omega \sim O(1)$, and find that $C_{T} \sim(\alpha-1)^{2 l+1}$, and that, for $l_{0}=0$, $\left|C_{T}\right| \approx(\alpha-1) \omega / 2$. Because the transmission factor $C_{T}$ is increasingly suppressed for increasing $l$, we will be mostly interested in the contribution from the $l_{0}=0$ mode, as the other contributions will be smaller than the terms that we neglect in our approximate calculation of the $l_{0}=0$ contribution.

The propagation from $H_{a l}^{+}$and $H_{a r}^{+}$to $\mathcal{I}^{+}$is also described in appendix B. This part of the calculation is substantially easier; it is very similar to solving the angular equation (35). In the conformal frame where the metric has the form (12), the restriction of $f_{\omega^{\prime} l m}^{b}$ to $\mathcal{I}^{+}$is

$$
\left.f_{\omega^{\prime} l m}^{b}\right|_{\mathcal{I}^{+}}=\frac{2 N C_{T} G^{1 / 2}(y)}{\left|1-e^{-2 \pi \omega^{\prime} / \kappa}\right|^{1 / 2}\left|G^{\prime}\left(\xi_{4}\right)\right| \sin \theta} e^{-i \omega^{\prime}(t+z)} e^{i m \varphi} e^{-m|z|} f_{l \omega^{\prime}}(p) \tilde{n}_{l m}(p) .
$$

In this expression, $f_{l \omega^{\prime}}(p)$ is given by the definition at the end of appendix $\mathrm{B}$, and we have defined $\tilde{n}_{l m}(p)$ to be $n_{l m}(p)$ for $p<1 / 2(\chi<0)$ and $e^{2 m \chi} n_{l m}(p)$ for $p>1 / 2(\chi>0)$, where $n_{l m}(p)$ is the approximate solution of the angular equation given in appendix A. When $p \rightarrow 0, f_{l \omega^{\prime}}(p), \tilde{n}_{l m}(p) \rightarrow 1$. When $p \rightarrow 1, \tilde{n}_{l m}(p) \rightarrow e^{i \varrho}$, some constant phase.

Evaluating the inner product, we find that

$$
\bar{\beta}_{\omega \omega^{\prime} l m}^{b}=-\frac{2 \bar{N} \bar{C}_{T} \omega e^{i \omega^{\prime} t_{0}} e^{-i m \varphi_{0}}}{\sqrt{2 \pi}\left|G^{\prime}\left(\xi_{4}\right)\right|\left|1-e^{-2 \pi \omega^{\prime} / \kappa}\right|^{1 / 2}} \int d z e^{i \omega r} e^{i \omega^{\prime} z} e^{-m|z|} f_{l \omega^{\prime}}(p) \tilde{n}_{l m}(p),
$$

where $t=t_{0}$ corresponds to $\theta=\theta_{0}, \varphi=\varphi_{0}$ corresponds to $\eta=\eta_{0}$, and we have used $d r=d z / G^{1 / 2}(y)$, which follows from (14) and (21).

Note that apart from an overall phase, this expression depends only on the frequency $\omega$, and not on $\theta_{0}, \eta_{0}$. This means that the expression is boost invariant, that is, invariant under translations in $t$, as the orbits of the boosts are the cross-sections labeled by $r$, and thus these boosts preserve the frequency $\omega$ with respect to $r$.

We can't evaluate the integral in (49), but we can still get some interesting physical information about the radiation out of this expression. Because $G(y) \rightarrow 0$ as $z \rightarrow \pm \infty$,

$$
\frac{d r}{d z}=\frac{1}{G^{1 / 2}(y)} \rightarrow \pm \infty \text { when } z \rightarrow \pm \infty,
$$

and hence the $e^{i \omega r}$ part of the integrand oscillates with an effective frequency which tends to infinity at large $|z|$. Since the amplitude is bounded, the main contribution to the integrand will come from the region near $z=0$ where the integrand oscillates slowly.

The integral in (49) will give an answer which is peaked in $\omega^{\prime}$ with some finite width, so the integration over $\omega^{\prime}$ of $\left|\bar{\beta}_{\omega \omega^{\prime} l m}^{b}\right|^{2}$ in (43) should give a finite answer. By contrast, in the case of a static black hole, the analogous formula for the Bogoliubov coefficient 
gives a delta function in $\omega^{\prime}$, so the expected number of particles is infinite (that is, in that case there is a steady flux of particles across $\mathcal{I}^{+}$).

Our calculation of the transmission factor in appendix B is only valid for $\left|\omega^{\prime}\right| \leq 1$, and we might expect that for sufficiently large $\omega^{\prime}$, the potential barrier would become unimportant, and $C_{T} \sim O(1)$. However, the Bogoliubov coefficient will be small for large negative $\omega^{\prime}$ because of the factor $\left|1-e^{-2 \pi \omega^{\prime} / \kappa}\right|^{-1 / 2}$. We also expect that it would be small at large positive $\omega^{\prime}$, as the integrand in the integral in (49) will then oscillate rapidly for all values of $z$, making the integral small. Thus, the main contribution to the integral over $\omega^{\prime}$ in (43) will come from small negative $\omega^{\prime}$, where the calculation of $C_{T}$ is valid.

We expect that the size of the contribution from each $l, m$ will be primarily determined by the transmission factor, so we expect that the contribution from $l_{0}=m_{0}=0$ will dominate the summation over $l, m$ in (43). We now consider the form of this contribution in the point-particle limit, where we can somewhat simplify the expressions and illustrate some of these remarks. When $(\alpha-1) \ll 1$, we have $G(y) \approx 4 p(1-p)$ on $\mathcal{I}^{+}$, where $p=(\hat{y}-1) /(\alpha-1)$. Further, $z \approx \frac{1}{2} \ln (p /(1-p))$, as $0 \leq p \leq 1$ on $\mathcal{I}^{+}$, so

$$
G(y) \approx \frac{1}{\cosh ^{2} z}
$$

Thus, $d r / d z \approx \cosh z$, and hence

$$
r \approx \sinh z .
$$

Also, $\kappa \approx 1, f_{0 \omega}(p) \approx 1$, and $\tilde{n}_{00}(p) \approx 1$. Therefore

$$
\bar{\beta}_{\omega \omega^{\prime} 00}^{b} \approx-\frac{\bar{N} \bar{C}_{T} \omega e^{i \omega^{\prime} t_{0}} e^{-i m \varphi_{0}}}{\sqrt{2 \pi}\left|1-e^{-2 \pi \omega^{\prime}}\right|^{1 / 2}} \int d z e^{i\left(\omega^{\prime} z+\omega \sinh z\right)} .
$$

As we argued above, the main contribution to the integration will come from the region near $z=0$, so the primary contribution to $\bar{\beta}_{\omega \omega^{\prime} 00}^{b}$, and hence to the number operator, will come from the part of the generator closest to the points where the black holes intersect $\mathcal{I}^{+}$. If we restrict our attention to the region near $z=0$, we can expand $\sinh z$ in a power series, and we see that the integrand is most nearly constant near $z=0$ if $\omega^{\prime}=-\omega$, so we expect that the Bogoliubov coefficient will be peaked at $\omega^{\prime}=-\omega$. This peak will become narrower as $\omega \rightarrow 0$, approaching a delta function in the limit, but the amplitude tends to zero in this limit because of the factor of $\omega$ in front of the integral, so this does not imply infinite particle production.

The leading-order part of the total particle production along the generator labeled by $\theta_{0}, \eta_{0}$ is given by integrating $\left|\beta_{\omega \omega^{\prime} 00}^{b}\right|^{2}$ over $\omega$ and $\omega^{\prime}$; we can't do this integral, but given the arguments above, it seems reasonable to expect the answer to be finite. The integration over all generators, which gives the total particle production, will not give rise to any divergences either. 


\section{Discussion}

In the first part of this paper, we argued that the scattering off virtual black hole pairs, which could lead to loss of quantum coherence in ordinary scattering processes, could be discussed in terms of a path integral over Euclidean metrics with topology $S^{2} \times S^{2}-\{$ point $\}$. In this approach, one considers the scattering in each metric and performs a path integral over all such metrics. Since we cannot perform this path integral, we then restricted the discussion to one such metric, and analytically continued the solution to a Lorentzian section to make the scattering easier to understand.

We argued that the appropriate quantum state is the analytically-continued Euclidean vacuum state $|0\rangle_{E}$, and we argued that this state will contain a finite, non-zero number of particles at infinity. It is well-known that from the point of view of an observer co-moving with the black holes, this state corresponds to a thermal equilibrium between the black holes and a thermal bath of acceleration radiation. Thus, this state must be time-reversal invariant, which means that the particle content at past null infinity $\mathcal{I}^{-}$is the time-reverse of the particle content at future null infinity $\mathcal{I}^{+}$. This implies that no net energy is gained or lost by the black holes in this scattering process, which is what we would expect for a model of a virtual loop, and is in agreement with the fact that the state is an equilibrium as seen by co-moving observers.

The fact that there is a non-zero number of particles at $\mathcal{I}^{+}$implies that there is loss of quantum coherence in this semi-classical calculation, as each particle detected at infinity can be thought of as one member of a virtual pair, the other one of which has fallen into the black hole, carrying away information. More formally, there are correlations between modes on future infinity and modes on the future black hole horizon, and the information encoded in these correlations is lost because we do not observe the state on the future black hole horizon. This loss of quantum coherence is of the same character as that observed in static black holes.

In the second part of the paper, we proceeded to an explicit calculation of the scattering in the $C$ metric. Although the Euclidean $C$ metric solution has topology $S^{2} \times S^{2}-\{$ point $\}$, it is not usually thought of as describing a virtual black hole loop, as it is a solution of the field equations, and it has a conical singularity along one of the axes. However, we believe it is a reasonably good model for a virtual black hole loop, and the wave equation separates in this background, so it is relatively easy to study the scattering explicitly. The $C$ metric is asymptotically flat [14], so it is also straightforward to study the radiation at infinity. One slightly surprising fact about the structure at infinity is that the affine parameter along generators of $\mathcal{I}^{+}$is $\tilde{r}$, which is spacelike between the black hole and acceleration horizons, while the boost time coordinate $t$ becomes a spacelike coordinate labeling the generators of $\mathcal{I}^{+}$.

It is also worth noting that the transmission factor $C_{T} \sim(\alpha-1)^{2 l+1}$. This implies that the dominant contribution to the particle production is in the s-wave, as for static 
black holes, because of the high centrifugal potential barrier for higher-spin modes. It also suggests that the scattering of higher-spin fields off such virtual black hole loops will be suppressed relative to that of scalar fields, as they cannot radiate in the s-wave. This is in agreement with the arguments of [20, 19].

The calculation we have actually been able to perform is rather limited; we considered only one specific, rather special metric, and we were only able to study the scattering on it in a particular limit. However, the results we have obtained give an estimate of the magnitude and nature of the effects of virtual black hole loops, and they agree well with our general expectations.

\section{Acknowledgements}

We thank Gary Horowitz and Don Marolf for useful discussions. SFR thanks St. John's College Cambridge and the Natural Sciences and Engineering Research Council of Canada for financial support. SFR's work was also supported in part by NSF Grant PHY95-07065.

\section{A The angular quantization condition}

In the point-particle limit $(\alpha-1) \ll 1$, the deviations from spherical symmetry in the $x, \varphi$ part of the metric become small, so we would expect that the dependence on $x$ will reduce to the usual angular momentum modes, with quantum numbers $l$ and $m$. Recall that because of the periodicity of $\varphi, m=m_{0}\left|G^{\prime}\left(\xi_{4}\right)\right| / 2=m_{0}[1+O(\alpha-1)]$, where $m_{0}$ is an integer. We also expand $l=l_{0}+l_{1}(\alpha-1)+\ldots$ The range of $\hat{x}$ is $1 \leq \hat{x} \leq \alpha$, so we define a new coordinate $p=(\hat{x}-1) /(\alpha-1)$. If we expand $\hat{n}(\hat{x})$ in powers of $\alpha-1$, $\hat{n}(\hat{x})=n_{l m}(p)=n_{0}(p)+(\alpha-1) n_{1}(p)+\ldots$, then (35) can be separated into a series of equations for these functions. The first equation is

$$
\partial_{p}\left[p(p-1) \partial_{p} n_{0}(p)\right]-m_{0} \partial_{p} n_{0}(p)-l_{0}\left(l_{0}+1\right) n_{0}(p)=0 .
$$

This equation is a hypergeometric equation. The possible values of $l_{0}$ are restricted by requiring that the solution behave appropriately at the two poles, $p=0,1$. As we said earlier, for the solution for $\phi$ to be physically relevant, we must have $\nu(x) \sim e^{-m|\chi|}$ as $\chi \rightarrow \pm \infty$. That is, we require that $\nu(x)$, and hence $\phi$, doesn't blow up at the axes. Since $\hat{\nu}(\hat{x})=e^{m \chi} \hat{n}(\hat{x})$, the appropriate boundary conditions on $n_{l m}(p)$ are that $n_{l m}(p)=1$ as $\chi \rightarrow-\infty$, which corresponds to $p=0$, and $n_{l m}(p) \sim e^{-2 m \chi}$ as $\chi \rightarrow \infty$, which corresponds to $p=1$. Therefore, the appropriate solution of the hypergeometric equation (54) is $n_{0}(p)=F\left(l_{0}+1,-l_{0} ; 1+m_{0} ; p\right)$, where $F$ is the hypergeometric series, 
as $F(a, b ; c ; p) \rightarrow 1$ as $p \rightarrow 0$. If we analytically continue this solution to a neighborhood of $p=1$, we find

$$
\begin{aligned}
n_{0}(p)= & \frac{\Gamma\left(1+m_{0}\right) \Gamma\left(m_{0}\right)}{\Gamma\left(m_{0}-l_{0}\right) \Gamma\left(m_{0}+l_{0}+1\right)} F\left(l_{0}+1,-l_{0}, 1-m_{0} ; 1-p\right) \\
& +\frac{\Gamma\left(1+m_{0}\right) \Gamma\left(-m_{0}\right)}{\Gamma\left(l_{0}+1\right) \Gamma\left(-l_{0}\right)}(1-p)^{m_{0}} F\left(m_{0}-l_{0}, 1+m_{0}+l_{0} ; 1+m_{0} ; 1-p\right) .
\end{aligned}
$$

The second term has the appropriate behavior for $p \rightarrow 1$, since $e^{-\chi} \approx(1-p)^{1 / 2}$ for $p \approx 1$. Thus, the coefficient of the first term must vanish, which can only happen if $l_{0}-m_{0}$ is a non-negative integer. This is just the usual quantisation condition for angular momentum, and $l_{0}$ is thus the total angular momentum quantum number.

The next-order term $l_{1}$ can similarly be fixed by requiring that the solution $n_{1}(p)$ is regular at $p=0,1$. Unfortunately, it is not possible to give a general formula for $l_{1}$; the equation must be solved separately for each $l_{0}, m_{0}$. We are particularly interested in the case $l_{0}=m_{0}=0$, as we expect this mode to make the dominant contribution to the particle production on $\mathcal{I}^{+}$. In this case, $n_{0}(p)=F(1,0 ; 1 ; p)=1$, while the equation for $n_{1}(p)$ is

$$
\partial_{p}\left[p(p-1) \partial_{p} n_{1}(p)\right]=l_{1}-p .
$$

This equation has a solution which is regular at $p=0,1$ only if $l_{1}=1 / 2$; in this case, the solution is $n_{1}(p)=-p / 2+C$, where $C$ is a constant. One can similarly fix all the $l_{i}$.

\section{B The transmission factor}

In section 6 , we found that to evolve the positive-frequency modes from $\mathcal{C}$ to $\tilde{\mathcal{C}}$, we need to calculate the transmission factor $C_{T}$ between the black hole and acceleration horizons. That is, we need to solve (36) with the boundary conditions $(46,47)$, and find $C_{T}$. For convenience, we will repeat those here. The equation is

$$
\partial_{\hat{y}}\left[\left(\hat{y}^{2}-1\right)\left(\hat{y}^{2}-\alpha^{2}\right) \partial_{\hat{y}} \hat{f}(\hat{y})\right]+2 i \omega \zeta \partial_{\hat{y}} \hat{f}(\hat{y})+2\left(\hat{y}^{2}-\beta_{D}^{2}\right) \hat{f}(\hat{y})=0 .
$$

In terms of the function $\hat{f}(\hat{y})$, the boundary conditions are

$$
\hat{f}(\hat{y})=1+C_{R} e^{2 i \omega z}
$$

near the black hole horizon $\hat{y}=-1$ and

$$
\hat{f}(\hat{y})=C_{T}
$$

near the acceleration horizon $\hat{y}=1$. 
We can't solve this equation exactly, but if $(\alpha-1) \ll 1$, then we can solve it approximately. First note that if $\hat{y}^{2}-1$ is $O(1)$ (that is, if $\hat{y}$ is not close to \pm 1 ), we can neglect terms involving $\alpha-1$ to approximate (57) as

$$
\partial_{\hat{y}}\left[\left(\hat{y}^{2}-1\right)^{2} \partial_{\hat{y}} \hat{f}(\hat{y})\right]+2\left(\hat{y}^{2}-\beta_{D}^{2}\right) \hat{f}(\hat{y})=0 .
$$

In neglecting the term involving $\omega$, we have made the further assumption that $|\omega| \sim O(1)$; that is, that $\omega$ is not large. This equation is now a hypergeometric equation. To put it in the standard form, we set $\hat{f}(\hat{y})=2^{a}\left(1-\hat{y}^{2}\right)^{-a}(\alpha-1)^{a} g(s)$, where $s=(\hat{y}+1) / 2$, $a=l+1$. Then

$$
s(s-1) \partial_{s}^{2} g(s)-2 l(2 s-1) \partial_{s} g(s)+2 l(2 l+1) g(s)=0,
$$

where we have used $\beta_{D}=1+2 l(l+1)$. We use $l$ rather than $l_{0}$ in the approximate equations in this section, because regarding $l$ as an integer would introduce degeneracies in the approximate equations which are not present in the exact equation. Near $\hat{y}= \pm 1$, the solutions of (61) can be expressed in terms of hypergeometric series about $\hat{y}= \pm 1$. However, we cannot approximate (57) by (61) in a neighborhood of radius $O(\alpha-1)$ around $\hat{y}= \pm 1$, which is precisely where we wish to impose boundary conditions.

Therefore we need a separate approximation to cover these neighborhoods. When $\hat{y}^{2}-1 \sim(\alpha-1)$, make a coordinate transformation $\hat{y}= \pm\left(1+(\alpha-1) q_{ \pm}\right)$. Then if we keep just the leading terms, (57) becomes

$$
\partial_{q_{ \pm}}\left[q_{ \pm}\left(q_{ \pm}-1\right) \partial_{q_{ \pm}} f\left(q_{ \pm}\right)\right] \pm i \omega \partial_{q_{ \pm}} f-l(l+1) f=0,
$$

where $f\left(q_{ \pm}\right)=\hat{f}(\hat{y})$. These are, once again, hypergeometric equations. The solution about $\hat{y}=-1$ which satisfies the boundary condition (58) is

$$
f\left(q_{-}\right)=F\left(a, b ; 2-c ; q_{-}\right)+C_{R}\left(-q_{-}\right)^{-i \omega} F\left(b+c-1, a+c-1 ; c ; q_{-}\right),
$$

and the solution about $\hat{y}=1$ which satisfies the boundary condition (59) is

$$
f\left(q_{+}\right)=C_{T} F\left(a, b ; c ; q_{+}\right),
$$

where $F$ is the hypergeometric function, $a=l+1, b=-l$ and $c=1-i \omega$. Now analytically extend these solutions to large $q_{ \pm}$: at large $q_{-}$, the solution (63) becomes

$$
\begin{aligned}
f\left(q_{-}\right)= & \frac{\Gamma(c) \Gamma(b-a)}{\Gamma(c-a) \Gamma(b)}\left(C_{R}+\frac{\Gamma(2-c) \Gamma(c-a)}{\Gamma(c) \Gamma(2-c-a)}\right)\left(-q_{-}\right)^{-a} \\
& +\frac{\Gamma(c) \Gamma(a-b)}{\Gamma(c-b) \Gamma(a)}\left(C_{R}+\frac{\Gamma(2-c) \Gamma(c-b)}{\Gamma(c) \Gamma(2-c-b)}\right)\left(-q_{-}\right)^{-b},
\end{aligned}
$$


while at large $q_{+}$, the solution (64) becomes

$$
f\left(q_{+}\right)=C_{T} \frac{\Gamma(c) \Gamma(b-a)}{\Gamma(c-a) \Gamma(b)}\left(-q_{+}\right)^{-a}+C_{T} \frac{\Gamma(c) \Gamma(a-b)}{\Gamma(c-b) \Gamma(a)}\left(-q_{+}\right)^{-b} .
$$

Now for $1 \ll\left|q_{ \pm}\right| \ll(\alpha-1)^{-1}$, both approximations are applicable, so we can use the large-distance behavior $(65,66)$ of the approximation for $\hat{y}$ near \pm 1 as boundary data for the approximation (61). If we pick the solution $g(s)$ to be

$$
\begin{aligned}
g(s)= & \frac{\Gamma(c) \Gamma(b-a)}{\Gamma(c-a) \Gamma(b)}\left(C_{R}+\frac{\Gamma(2-c) \Gamma(c-a)}{\Gamma(c) \Gamma(2-c-a)}\right) F(-2 l,-2 l-1 ;-2 l ; s) \\
& +\frac{\Gamma(c) \Gamma(a-b)}{\Gamma(c-b) \Gamma(a)}\left(C_{R}+\frac{\Gamma(2-c) \Gamma(c-b)}{\Gamma(c) \Gamma(2-c-b)}\right)(\alpha-1)^{b-a} 2^{a-b} s^{a-b} F(0,1 ; 2 l+2 ; s),
\end{aligned}
$$

then the boundary conditions obtained from (65) are automatically satisfied. We can analytically continue this solution to a neighborhood of $s=1$; to satisfy the boundary conditions obtained from (66) in this neighborhood at the same time, we must require

$$
C_{T} \frac{\Gamma(c) \Gamma(b-a)}{\Gamma(c-a) \Gamma(b)}=\frac{\Gamma(c) \Gamma(a-b)}{\Gamma(c-b) \Gamma(a)}\left(C_{R}+\frac{\Gamma(2-c) \Gamma(c-b)}{\Gamma(c) \Gamma(2-c-b)}\right)(\alpha-1)^{b-a} 2^{a-b}
$$

and

$$
C_{T} \frac{\Gamma(c) \Gamma(a-b)}{\Gamma(c-b) \Gamma(a)}(\alpha-1)^{b-a} 2^{a-b}=\frac{\Gamma(c) \Gamma(b-a)}{\Gamma(c-a) \Gamma(b)}\left(C_{R}+\frac{\Gamma(2-c) \Gamma(c-a)}{\Gamma(c) \Gamma(2-c-a)}\right) .
$$

Solving these two equations for $C_{R}$ and $C_{T}$, we find

$$
C_{T}=-e^{i \vartheta} \frac{\delta-\bar{\delta}}{1-\delta^{2}}
$$

and

$$
C_{R}=-e^{i \vartheta}+\delta C_{T},
$$

where

$$
e^{i \vartheta}=\frac{\Gamma(2-c) \Gamma(c-b)}{\Gamma(c) \Gamma(2-c-b)}
$$

and

$$
\delta=\left(\frac{\alpha-1}{2}\right)^{a-b} \frac{\Gamma(b-a) \Gamma(a) \Gamma(c-b)}{\Gamma(a-b) \Gamma(b) \Gamma(c-a)} .
$$

Note that these coefficients satisfy $\left|C_{T}\right|^{2}+\left|C_{R}\right|^{2}=1$, as they should. 
After some manipulation, we find

$$
\delta-\bar{\delta}=-\frac{4 i}{2 l+1}\left(\frac{\alpha-1}{8}\right)^{2 l+1} \frac{\Gamma(1+l-i \omega) \Gamma(1+l+i \omega)}{\Gamma\left(l+\frac{1}{2}\right)^{2}} \sinh \pi \omega .
$$

Also, $\delta \sim(\alpha-1)^{2 l+1}$, so the denominator in $C_{T}$ can be ignored for this leading-order calculation. For large $l$, we thus find

$$
C_{T} \approx 2 e^{i\left(\vartheta+\frac{\pi}{2}\right)}\left(\frac{\alpha-1}{8}\right)^{2 l+1} \sinh \pi \omega
$$

while for $l_{0}=0$, we find

$$
C_{T} \approx e^{i\left(\vartheta+\frac{\pi}{2}\right)}\left(\frac{\alpha-1}{2}\right) \omega .
$$

These results are valid for $(\alpha-1) \ll 1$ and $|\omega| \leq 1$.

We have found the value of $\hat{f}(\hat{y})$ at the acceleration horizon $\hat{y}=1$. The region between $H_{a l}^{+}, H_{a r}^{+}$, and $\mathcal{I}^{+}$is the region between $\hat{y}=1$ and $\hat{y}=\hat{x}$; to evolve $\hat{f}(\hat{y})$ through this region, we just need to find the form of $\hat{f}(\hat{y})$ between $\hat{y}=1$ and $\hat{y}=\alpha$, which will also be the solution on $\mathcal{I}^{+}$. Now, the approximation (64) is valid throughout this region, so the result is simply that on $\mathcal{I}^{+}$,

$$
\hat{f}(\hat{y})=C_{T} f_{l \omega}(p) \approx C_{T} F(a, b ; c ; p),
$$

where $a, b, c$ are as in (64). Note that $\hat{x}=\hat{y}$ implies $q_{+}=p$. For $l_{0}=0$, the leading-order part of this solution is $f_{0 \omega}(p) \approx 1$, just as for $n_{l m}(p)$.

\section{References}

[1] A. Strominger and C. Vafa, "Microscopic origin of the Bekenstein-Hawking entropy," Phys. Lett. B379, 99 (1996), hep-th/9601029.

[2] G. T. Horowitz, "The origin of black hole entropy in string theory," gr-qc/9604051.

[3] J. M. Maldacena, "Black holes in string theory," hep-th/9607235.

[4] S. R. Das and S. D. Mathur, "Comparing decay rates for black holes and d-branes," Nucl. Phys. B478, 561 (1996), hep-th/9606185.

[5] J. Maldacena and A. Strominger, "Black hole grey body factors and d-brane spectroscopy," Phys. Rev. D 55, 861 (1997), hep-th/9609026. 
[6] S. W. Hawking, "The unpredictability of quantum gravity," Commun. Math. Phys. 87, 395 (1982).

[7] S. W. Hawking, "Virtual black holes," Phys. Rev. D 53, 3099 (1996), hep-th/9510029.

[8] J. B. Hartle and S. W. Hawking, "Path-integral derivation of black-hole radiance," Phys. Rev. D 13, 2188 (1976).

[9] P. Yi, "Vanishing Hawking radiation from a uniformly accelerated black hole," Phys. Rev. Lett. 75, 382 (1995), gr-qc/9503047.

[10] P. Yi, "Quantum stability of accelerated black holes," Phys. Rev. D 53, 7041 (1996), hep-th/9505021.

[11] D. N. Page, "Particle emission rates from a black hole: Massless particles from an uncharged, nonrotating hole," Phys. Rev. D 13 (1976).

[12] W. Kinnersley and M. Walker, "Uniformly accelerating charged mass in general relativity," Phys. Rev. D 2, 1359 (1970).

[13] F. Dowker, J. P. Gauntlett, D. A. Kastor, and J. Traschen, "Pair creation of dilaton black holes," Phys. Rev. D 49, 2909 (1994), hep-th/9309075.

[14] A. Ashtekar and T. Dray, "On the existence of solutions to Einstein's equation with nonzero Bondi news," Commun. Math. Phys. 79, 581 (1981).

[15] S. W. Hawking and S. F. Ross, "Pair production of black holes on cosmic strings," Phys. Rev. Lett. 75, 3382 (1995), gr-qc/9506020.

[16] R. Gregory and M. Hindmarsh, "Smooth metrics for snapping strings," Phys. Rev. D 52, 5598 (1995), gr-qc/9506054.

[17] D. M. Eardley, G. T. Horowitz, D. A. Kastor, and J. Traschen, "Breaking cosmic strings without monopoles," Phys. Rev. Lett. 75, 3390 (1995), gr-qc/9506041.

[18] R. M. Wald, Quantum Field Theory in Curved Spacetime and Black Hole Thermodynamics. Chicago University Press, Chicago, USA, 1994.

[19] S. W. Hawking, D. N. Page, and C. N. Pope, "Quantum gravitational bubbles," Nucl. Phys. B170, 283 (1980).

[20] S. W. Hawking, D. N. Page, and C. N. Pope, "The propagation of particles in space-time foam," Phys. Lett. 86B, 175 (1979). 